\title{
Pion and kaon SIDIS multiplicities from COMPASS
}

\author{
Fabienne Kunne ${ }^{1}$ \\ CEA IRFU \\ CEA Saclay, IRFU DPhN, F91190, France \\ E-mail: fabienne.kunnelcea.fr
}

\section{On behalf of the COMPASS Collaboration}

We present preliminary COMPASS results on pion and kaon multiplicities produced in semiinclusive deep inelastic scattering of $160 \mathrm{GeV}$ muons off an isoscalar ( $\left.{ }^{6} \mathrm{LiD}\right)$ target. The results constitute an impressive data set of more than 600 points for pions and 600 for kaons, covering a large $x, Q^{2}$ and $z$ domain in a fine binning. The data are available for QCD fits to extract quark fragmentation functions. We show results of a first leading order fit performed to extract the favored and unfavored quark fragmentation functions into pions $D_{\text {fav }}{ }^{\pi}$ and $D_{\text {unfav }}{ }^{\pi}$. The results from the sum of the $z$-integrated multiplicities $\mathrm{M}\left(\pi^{+}\right)+\mathrm{M}\left(\pi^{-}\right)$and $\mathrm{M}\left(\mathrm{K}^{+}\right)+\mathrm{M}\left(\mathrm{K}^{-}\right)$are presented versus $x$ and compared to previous experiments. At high $x$, the kaon data indicate a value of the nonstrange quark fragmentation function significantly larger than obtained by the earlier QCD DSS fit. Overall, the new kaon data will strongly impact the flavour decomposition of kaon fragmentation functions. Recent additional data were added at high $z$ on the ratio of $\mathrm{K}^{-}$to $\mathrm{K}^{+}$ multiplicities. For $z>0.8$, the ratio falls well below expectations from LO and NLO pQCD calculations. The ratio also shows a strong and unexpected dependence on the missing mass of the single kaon production process. This suggest to take into account the phase space available for hadronisation in further theoretical calculations. 


\section{Introduction}

The goal of the present measurements is to help determining parton fragmentation function. Parton fragmentation functions ( $\mathrm{FFs}$ ), like parton distribution functions (PDFs), are non perturbative objects. They describe the hadronization of partons. The FFs are process independent and are used to describe the probability that a quark of flavor q fragments into a hadron of type $\mathrm{h}$ $\left(D_{\mathrm{q}}^{\mathrm{h}}\right)$. They are needed e.g. for the extraction of flavor dependent quark polarization distributions $\Delta \mathrm{q}(x)[1,2]$ extracted from semi-inclusive polarized deep inelastic scattering. In particular, to access the strange quark polarization $\Delta \mathrm{s}$ from polarized SIDIS, the strange quark FF into kaon $D_{\mathrm{s}}{ }^{\mathrm{K}}$ is needed and it constitutes by far the largest contribution to the uncertainty in the extraction. Data sensitive to FFs exist from $\mathrm{e}^{+} \mathrm{e}^{-}$and $\mathrm{p}$ pbar reactions, but they are unsufficient for a good flavor separation; they also lie at relatively too high $Q^{2}$ values. This is why it was decided to measure charged hadron multiplicities in SIDIS with a high precision and a fine binning in several variables. The obtained multiplicities constitute inputs for the QCD fits to FFs.

\section{Charged pion and kaon multiplicities from SIDIS}

The hadron multiplicities $\mathrm{M}\left(x, Q^{2}, z\right)$ are defined as the mean number of hadrons $\mathrm{h}$ produced in a SIDIS event $\mu \mathrm{p} \rightarrow \mu \mathrm{hX}$. At leading order (LO), they are simply related to PDFs and quark FFs, as shown in the equation below. Here where $x$ is the fraction of momentum carried by the struck quark, $Q^{2}$ is the momentum transfer and $z$ is the energy fraction taken by the hadron

$$
M\left(x, Q^{2}, z\right)=\frac{\sum_{q} e_{q}^{2} q\left(x, Q^{2}\right) D_{q}^{h}\left(z, Q^{2}\right)}{\sum_{q} e_{q}^{2} q\left(x, Q^{2}\right)} .
$$

Note that apart from the $\mathrm{Q}^{2}$ dependence, PDFs depend upon $\mathrm{x}$, while FFs depend upon $z$. This will help to disentangle FFs from PDFs, while only the product of both is measured.

The $\pi$ and K multiplicities were calculated using COMPASS SIDIS data taken in 2006 with an isoscalar target $\left({ }^{6} \mathrm{LiD}\right)$ [3]. They were corrected by the value of the global acceptance of the apparatus estimated in each $(x, y, z)$ bin. The acceptance was evaluated from a Monte Carlo simulation, comparing the number of generated and reconstructed events in the whole chain of analysis. For this study, the $y$ variable is chosen instead of the $Q^{2}$ one, since $Q^{2}$ is too much correlated to $x$. For the $\pi$ and $\mathrm{K}$ identification a RICH detector is used. Additional corrections including RICH unfolding, radiative corrections and contributions from diffractive vector meson production were applied. Final $\pi$ and $\mathrm{K}$ multiplicities are published in [4,5].

We study first the pion case. Starting from the equation given above at LO, FFs can be extracted once PDFs are taken from the literature. In each $(x, y, z)$ bin, two equations are given by the $\pi^{+}$and $\pi^{-}$multiplicity measured in that bin. We can extract two FFs, namely the favored and unfavored quark FFs into pions. They correspond to valance and sea quarks respectively. They are the two independent FFs left after applying isospin and charge symmetry relations between FFs, and assuming in addition that the strange quark FF is equal to the unfavored one. In order to use simultaneously all data measured at various $Q^{2}$, a LO fit is performed. For this, functional forms are chosen for the shape of the two FFs at an initial $Q_{0}{ }^{2}$ value, and the DGLAP equations are used for the evolution to the measured $Q^{2}$ of each data point. Results for the favored and unfavored quark FF into pions obtained from the fit of the pion multiplicities are shown in Fig.1. The statistical error band was obtained with a replica method, varying the data points within their 
error. Our result [4] is in fair agreement with fits realized at NLO on similar SIDIS data [6]. They differ, as expected from fits including only $\mathrm{e}^{+} \mathrm{e}^{-}$data [7].
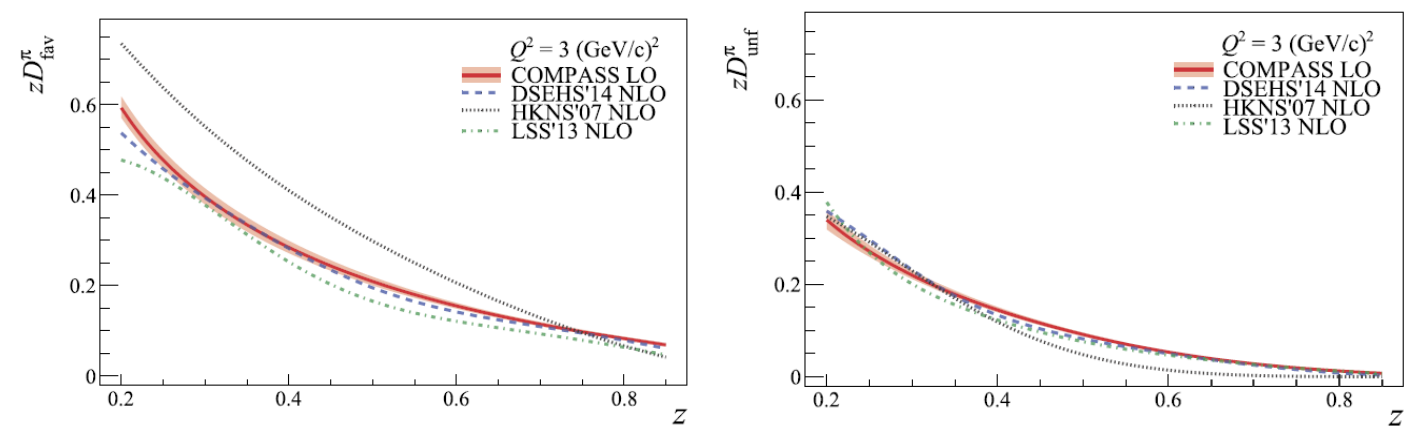

Fig.1 Favored (left) and unfavored (right) quark fragmentation functions into pions, from a LO fit to COMPASS $\pi$ multiplicities [3]

\section{Sum of z-integrated multiplicities $M\left(\pi^{+}\right)+M\left(\pi^{-}\right)$and $M\left(K^{+}\right)+M\left(K^{-}\right)$}

For an isoscalar target, assuming only two independent FFs for pions, $D_{\mathrm{fav}}{ }^{\pi}$ and $D_{\text {unfav }}{ }^{\pi}$, the sum of $z$-integrated multiplicities $\mathrm{M}\left(\pi^{+}\right)+\mathrm{M}\left(\pi^{-}\right)$has a simple expression at LO [4]. It is expected to be flat in $x$. Fig. 2 left shows the result for the sum integrated over $z$ from 0.2 to 0.85 and averaged over $y$ between 0.1 and 0.7 , as a function of $x$. The expected weak dependence in $x$ is observed in COMPASS data. The HERMES multiplicities, also shown in Fig.2, are larger. Note however that they are measured at a lower energy and correspond to different kinematics.
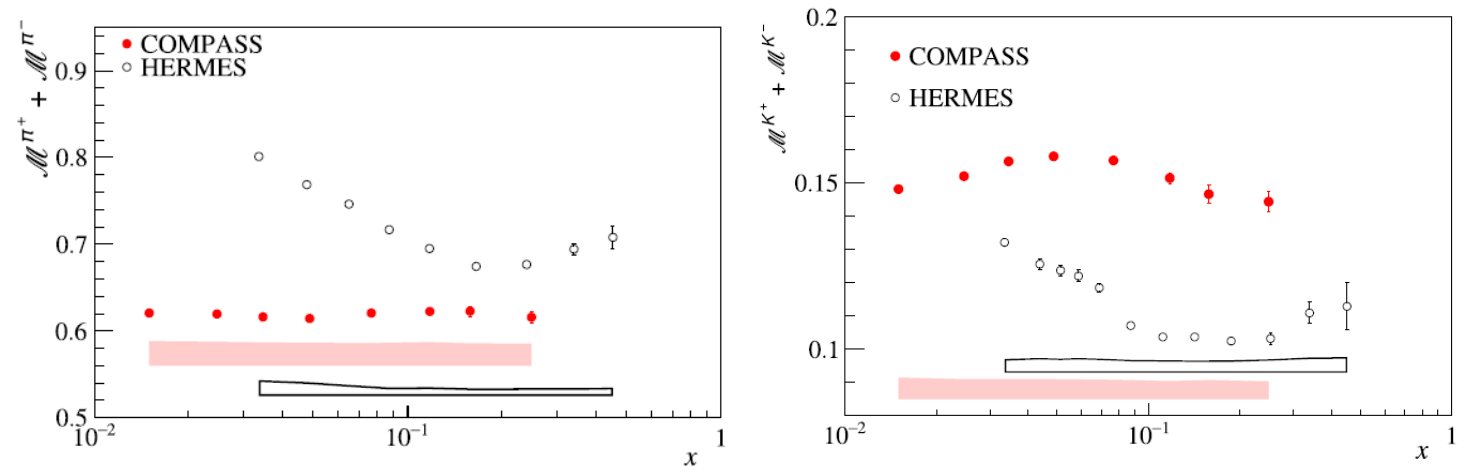

Fig.2 Sum of $z$-integrated multiplicities, for pions (left) and for kaons (right) versus $x$. COMPASS data (160 GeV, red full points) [3, 4] are compared to HERMES ones (27 GeV, black open points) [8].

For kaons, assuming three independent FFs, $D_{\text {fav }}{ }^{\pi}, D_{\text {unfav }}{ }^{\pi}$, and $D_{\text {str }}{ }^{\pi}$, the sum of $z$-integrated multiplicities $\mathrm{M}\left(\mathrm{K}^{+}\right)+\mathrm{M}\left(\mathrm{K}^{-}\right)$has also a simple expression at LO [4]. At high $x$, the strange content of the nucleon can be neglected, and the sum is sensitive to $D_{\mathrm{u}}{ }^{\mathrm{K}}$. At low $x$, the sum is sensitive to the product of the strange PDF and FF. The result for the sum of $z$-integrated multiplicities $\mathrm{M}\left(\mathrm{K}^{+}\right)$ 
$+\mathrm{M}\left(\mathrm{K}^{-}\right)$is shown in Fig.2 right. A weak $x$ dependence is observed. COMPASS data are situated well above the HERMES ones which cover different kinematics, in particular accepting lower $W$ values. From the COMPASS result on $\mathrm{M}\left(\mathrm{K}^{+}\right)+\mathrm{M}\left(\mathrm{K}^{-}\right)$, we extract $D_{\mathrm{u}}{ }^{\mathrm{K}} \approx 0.65-0.70$. This differs from earlier DSS fit at $Q^{2}=3(\mathrm{GeV} / \mathrm{c})^{2}, D_{\mathrm{u}}{ }^{\mathrm{K}} \approx 0.43(4)$ [6] mainly based on preliminary HERMES data which differ from the published ones [8].

The discrepancies between COMPASS and HERMES results have been further studied. While the kinematics between the two experiments differ significantly, we observe that for the very few points that have exactly the same kinematics in $x, y$ and $z$ variables, HERMES and COMPASS agree. For what concerns kaon data, calculations by Accardi et al. [9] show that target hadron mass corrections could explain part of the discrepancies. For what concerns pion data, where the discrepancy versus HERMES is reversed with lower values for COMPASS, simple Monte-Carlo calculations at LO suggest that different $W$ values could explain part of the overall shift in magnitude of the pion multiplicities, but not the different behavior vs $x$. More studies are needed to conclude.

\section{High z studies: ratio of $\mathrm{K}^{-}$to $\mathrm{K}^{+}$multiplicities}

Concerning the multiplicity data, the high $z$ region had not been studied so far. Recently, COMPASS published additional data [10] on studies of kaon production in SIDIS for kaons carrying a large fraction $z$ of the virtual photon energy. For this study only the ratio $R_{\mathrm{K}}$ of $\mathrm{K}^{-}$to $\mathrm{K}^{+}$multiplicities is studied. In the ratio, most experimental and theoretical uncertainties cancel. The kinematic domain requires $Q^{2}>1(\mathrm{GeV} / \mathrm{c})^{2}$ and $W>5$ $\mathrm{GeV} / \mathrm{c}^{2}$. The results are restricted to $z>0.75$, which leads to an effective upper limit for $y$ of 0.35 . The data are calculated in two $x$ bins, $\langle x\rangle=0.030$ and $\langle x\rangle=0.094$, as a function of different variables.

Fig. 3 shows the ratio $R_{\mathrm{K}}$ of $\mathrm{K}^{-}$to $\mathrm{K}^{+}$multiplicities, in the first $x$ bin, as a function of $z$. The data fall well below the expectations from several pQCD calculations at LO and NLO, especially for the highest $z$ values.

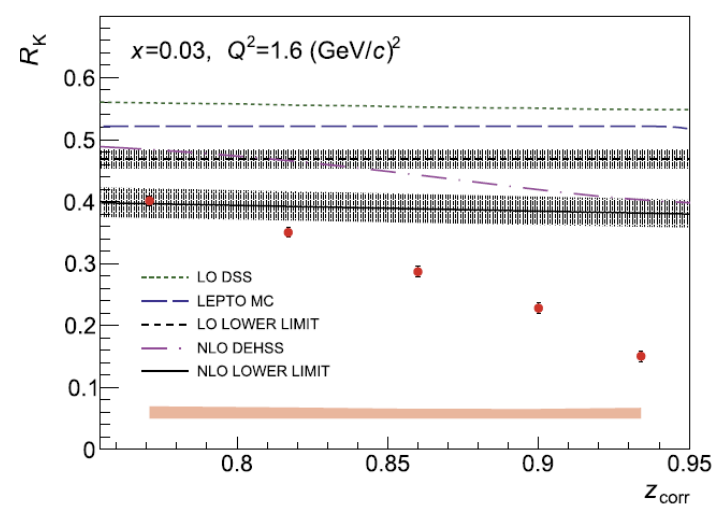

Fig.3 Ratio $R_{\mathrm{K}}$ of $\mathrm{K}^{-}$to $\mathrm{K}^{+}$multiplicities [10], in the first $x$ bin, as a function of $z$, compared to pQCD expectations or limits at LO and NLO. 
In Fig.4, the ratio $R_{\mathrm{K}}$ is shown as a function of $v$, the virtual photon energy. The data correspond to the same $x$ bin as above, $\langle x\rangle=0.03$. The five plots correspond to five $z$ bins. A clear linear $v$-dependence is observed except for the last $z$ bin. At larger values of $v$, the results are closer to the $\mathrm{pQCD}$ lower limit expectations.
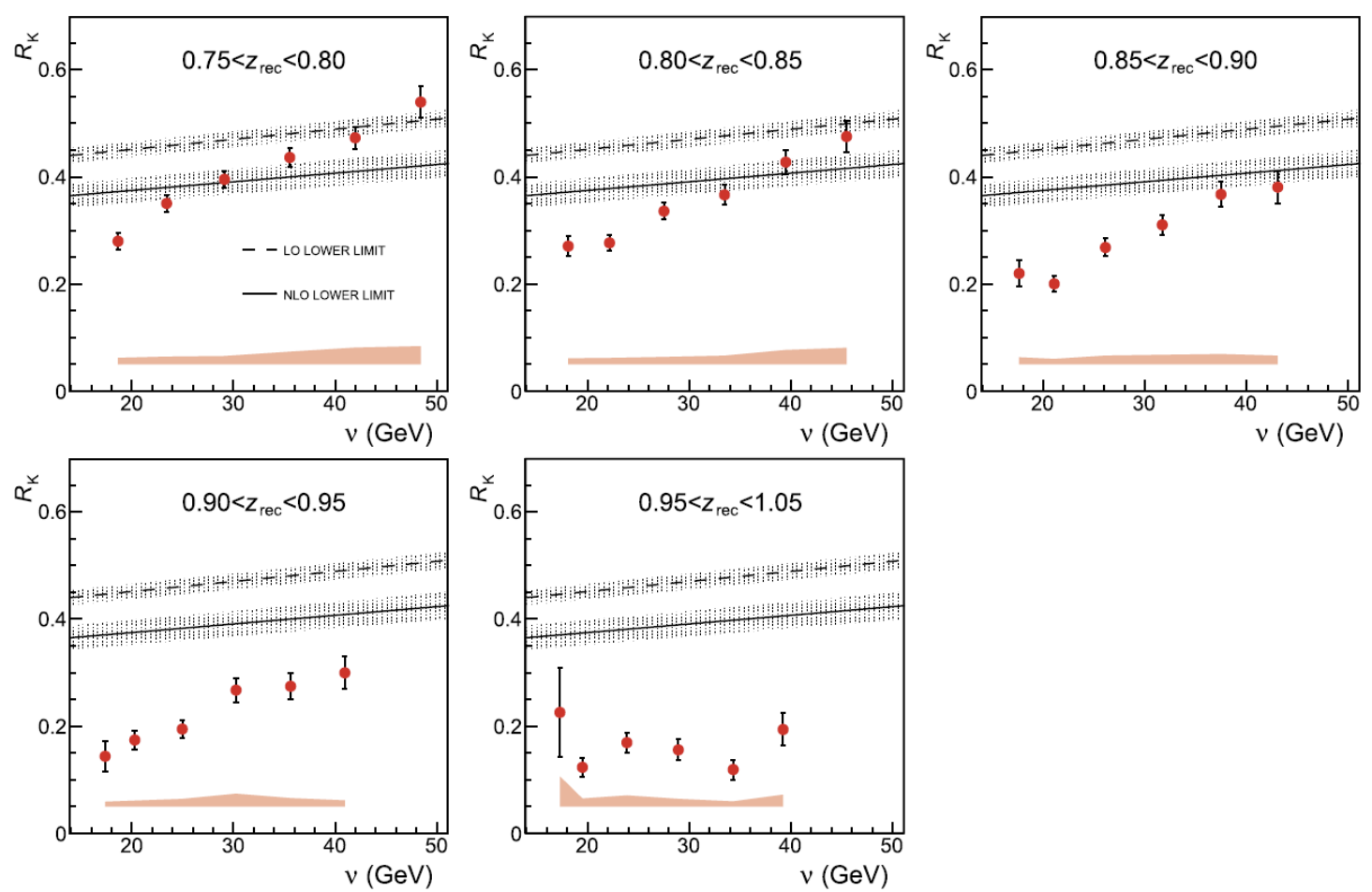

Fig.4 Ratio $R_{\mathrm{K}}$ of $\mathrm{K}^{-}$to $\mathrm{K}^{+}$multiplicities [10], in the first $x$ bin, as a function of $v$, compared to pQCD expected lower limits. The five boxes correspond to five $z$ bins.

The discrepancy observed with pQCD expectations, which is larger at large $z$ and small $y$, i.e., small $v$, can be interpreted as follows. At large $z$, only a small amount of energy is left to fulfil conservation laws such as strangeness or baryon number, which are not taken into account in the calculations. To further understand, we study the ratio $R_{\mathrm{K}}$ as a function of the missing mass $M_{\mathrm{X}}$ of the single kaon production process, defined as $M_{\mathrm{X}}{ }^{2}=M_{\mathrm{p}}{ }^{2}+$ $2 M_{\mathrm{p}} v(1-z)-Q^{2}(1-z)^{2}$. Fig. 5 shows an unexpected strong rise of $R_{\mathrm{K}}$ with $M_{\mathrm{X}}$, while no $M_{\mathrm{X}}$ dependence is foreseen in $\mathrm{pQCD}$ predictions. This suggests that a correction to the $\mathrm{pQCD}$ formalism is needed to take into account the available phase space for hadronization. As explained in [10], the results also suggest that the disagreement with pQCD may already be observed at smaller values of $z$ for experiments covering lower $v$ values. 


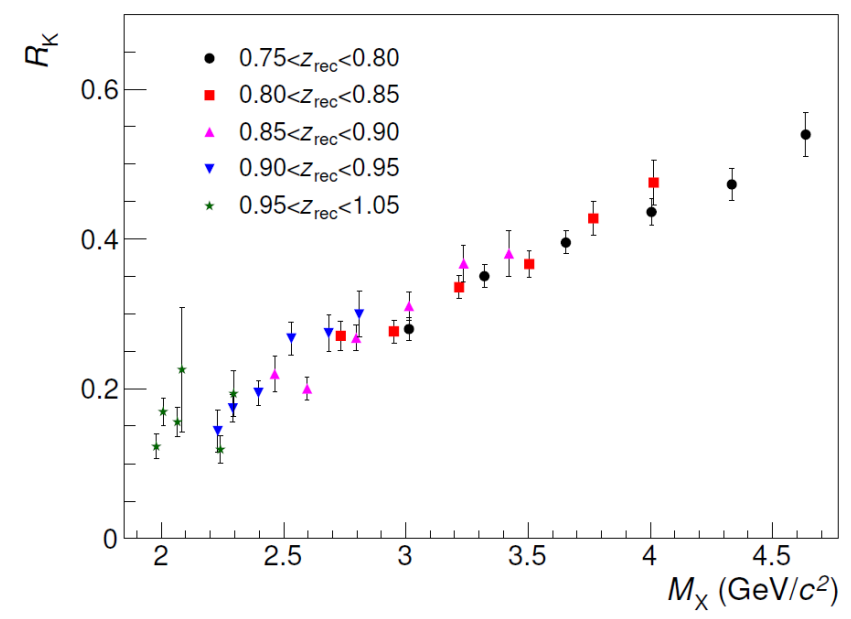

Fig.5. Ratio $R_{\mathrm{K}}$ of $\mathrm{K}^{-}$to $\mathrm{K}^{+}$multiplicities, in the first $x$ bin, as a function of $M_{\mathrm{x}}$.

\section{References}

[1] M. G. Alekseev et al., COMPASS Collab., Phys. Lett. B693 (2010) 227

[2] M. G. Alekseev et al., COMPASS Collab., Phys. Lett. B680 (2009) 217

[3] P. Abbon et al., COMPASS Collab., NIM A 517, 455 (2007).

[4] C.Adolph et al, COMPASS Collab., Phys. Lett. B764 (2017) 1

[5] C.Adolph et al, COMPASS Collab., Phys. Lett. B767 (2017) 133

[6] D. de Florian, R. Sassot and M. Stratmann, Phys. Rev. D 75, 114010 (2007)

[7] M. Hirai, S. Kumano, T.-H. Nagal and K. Sudoh, Phys. Rev. D 75,094009 (2007)

[8] HERMES Collab., A. Airapetian et al., PRD 89 (2014) 097101

[9] J. Guerrero and A. Accardi, PRD 97 (2018) 114012

[10] R. Akhunzyanov et al, COMPASS Collab., Phys. Lett. B786 (2018) 390 